\title{
Efficient Ex Vivo Expansion of $\gamma \delta$ T-Cells from AML Patients Requires Elimination of Circulating Leukemic Blasts
}

\author{
Ana C Parente-Pereira ${ }^{1}$, Pramila Krishnamurthy ${ }^{2}$, Lynsey M Whilding ${ }^{1}$, Kyriaki loannou ${ }^{3}$, \\ Sara Ciprut ${ }^{1}$, Victoria Potter ${ }^{2}$, Ghulam J Mufti ${ }^{2,3}$, Linda Barber ${ }^{3}$, Farzin Farzaneh ${ }^{3}$ and John \\ Maher $^{1,4,5,6^{*}}$
}

${ }^{1}$ King's College London, School of Cancer and Pharmaceutical Sciences, Guy's Hospital, Great Maze Pond, London SE1 9RT, UK

${ }^{2}$ Department of Haematological Medicine, King's College Hospital, Denmark Hill, London SE5 9RS, UK

${ }^{3}$ King's College London, School of Cancer and Pharmaceutical Sciences, The Rayne Institute, London SE5 9NU, UK

${ }^{4}$ Department of Immunology, Eastbourne Hospital, Kings Drive, Eastbourne, East Sussex, BN21 2UD, UK

${ }^{5}$ Department of Clinical Immunology and Allergy, King's College Hospital NHS Foundation Trust, Denmark Hill, London SE5 9RS, UK

${ }^{6}$ Leucid Bio Ltd., Guy's Hospital, Great Maze Pond, London SE1 9RT, UK

\begin{abstract}
$\delta$ T-cells play an important role in immune surveillance of acute myeloid leukemia (AML). The main circulating subtype expresses a $\mathrm{V} y 9 \mathrm{~V} \delta 2 \mathrm{~T}$-cell receptor and may be expanded ex vivo following aminobisphosphonate activation. While such protocols operate robustly in healthy donors, they are often inefficient using blood samples from patients with advanced malignancy. In keeping with this, we found that when leukemic blasts were present in peripheral blood samples, culture of $\gamma \delta$ T-cells from patients with AML was unsuccessful. By contrast, expansion proved much more effective after chemotherapy-mediated clearance of circulating blasts, yielding similar $\gamma \delta$ T-cell numbers to healthy donors. This principle was confirmed using serial samples obtained from the same patients. Importantly, expanded $\gamma \delta$ T-cells were functional, indicated by bisphosphonate-potentiated anti-leukemic activity and cytokine release in vitro. These preliminary findings suggest a therapeutic potential for $\gamma \delta$ T-cells, given that they can be readily expanded from AML patients in therapyinduced remission.
\end{abstract}

Keywords

Leukemia, Adoptive immunotherapy, $\gamma \delta$ t-cell, Zoledronic acid

\section{Introduction}

$\delta$ T-cells account for up to $3 \%$ of peripheral blood mononuclear cells (PBMC). In man, most express the $\mathrm{V} 9 \mathrm{~V} \delta 2$ receptor, enabling their MHC-independent activation by phosphoantigen (PAg) intermediates of the mevalonate pathway [1]. Dysregulation of mevalonate pathway metabolism is prevalent in AML [2], accounting for the frequent sensitivity of leukemic cells to statins [3] or aminobisphosphonates (e.g. alendronic and zoledronic acid) [4].

Several clinical observations demonstrate a role for $\gamma \delta$ T-cells in immune surveillance of AML [5]. First, prolonged survival of AML patients after allogeneic hematopoietic stem cell transplantation (HSCT) correlates with enhanced $\gamma \delta$ T-cell recovery. Second, these cells may be expanded ex vivo fol- lowing $\alpha \beta$ T-cell-depleted haploidentical HSCT and exhibit potent cytolytic activity against primary AML cells. Third, infusion of haploidentical $\gamma \delta$ T-cells followed by administration of zoledronic acid and IL-2 has induced complete remission

*Corresponding author: Department of Research Oncology, Guy's Hospital, Great Maze Pond, London SE1 9RT, UK, Tel: 0044-207-188-1468; Fax: 0044-207-188-0919

Accepted: December 26, 2020

Published online: December 28, 2020

Citation: Parente-Pereira AC, Krishnamurthy $P$, Whilding $L M$, et al. (2020) Efficient Ex Vivo Expansion of $\gamma \delta$ T-Cells from AML Patients Requires Elimination of Circulating Leukemic Blasts. Advances Leuk Res Treat 2(1):28-33 
Citation: Parente-Pereira AC, Krishnamurthy P, Whilding LM, et al. (2020) Efficient Ex Vivo Expansion of $\gamma \delta$ T-Cells from AML Patients Requires Elimination of Circulating Leukemic Blasts. Advances Leuk Res Treat 2(1):28-33

Table 1: Clinical information pertaining to samples tested.

\begin{tabular}{|c|c|c|c|c|c|}
\hline $\begin{array}{l}\text { Patient } \\
\text { Number }\end{array}$ & Clinical information & Age & Prior treatment & Interval post CTX (days) & Circulating blasts \\
\hline 001 - sample 1 & M1 (FLT3 ITD-) & 42 & $\mathrm{Nil}$ & No CTX & Yes \\
\hline 001 - sample 2 & & & $\mathrm{DA} \times 1$ & 17 & No \\
\hline 001 - sample 3 & & & $\mathrm{DA} \times 1$ & 42 & $\mathrm{No}^{*}$ \\
\hline & & & FLAG IDA $\times 2$ & & \\
\hline 002 - sample 1 & M1 (FLT3 ITD+ NPM+) & 19 & Nil & No CTX & Yes \\
\hline 002 - sample 2 & & & $\mathrm{DA} \times 1$ & 21 & No \\
\hline 003 - sample 1 & $\begin{array}{l}\text { Transformed from JAK2 mutant } \\
\text { myeloproliferative disorder }\end{array}$ & N/A & FLA $\times 1$ & 0 & No \\
\hline 003 - sample 2 & & & $\mathrm{FLA} \times 1$ & 26 & Yes \\
\hline 004 - sample 1 & M4 (Inv16) & 23 & $\mathrm{HU}$ & 0 & No \\
\hline 004 - sample 2 & & & $\mathrm{HU}+\mathrm{DA} \times 1$ & 26 & No \\
\hline 005 - sample 1 & $\begin{array}{l}\text { Transformed from refractory anemia } \\
\text { with excess blasts with Inv (3)(q21q26) }\end{array}$ & N/A & DA & 46 & No \\
\hline 005 - sample 2 & & & $\mathrm{DA} \times 2$ & 15 & No \\
\hline 006 & M1 FLT3 ITD+ & 41 & $\mathrm{DA} \times 1$ & 28 & No \\
\hline 007 - sample 1 & M1 FLT3 ITD+ & 56 & $\mathrm{DA} \times 1$ & 25 & No \\
\hline 007 - sample 2 & & & $\mathrm{DA} \times 2$ & 28 & No \\
\hline 008 & M4 t(6;11)(q27;q23) & 22 & $\mathrm{DA} \times 1$ & 28 & No \\
\hline 009 & $\begin{array}{l}\text { Transformed from JAK2 mutant } \\
\text { myeloproliferative disorder; trisomy } 8\end{array}$ & 57 & $\mathrm{Nil}$ & No CTX & Yes \\
\hline 010 & N/A & N/A & Nil & No CTX & Yes \\
\hline
\end{tabular}

CTX - chemotherapy; DA - daunorubicin + cytarabine; FLA - fludarabine + cytarabine; FLAG-IDA - fludarabine, cytarabine, G-CSF, idarubicin; $\mathrm{HU}$ - hydroxyurea; N/A - not available;

*Absolute lymphocyte count $0.2 \times 10^{9} / \mathrm{L}$ with no $\mathrm{CD}^{+}$cells detectable. Consequently, no expansion of $\mathrm{y} \delta$ T-cells was observed from this sample.

in patients with advanced hematologic malignancy, including AML. Fourth, in vivo activation of these cells with zoledronic acid and IL-2 has achieved an efficacy signal in AML patients.

These findings provide a rationale to develop $\gamma \delta$ T-cellbased immunotherapeutic strategies for AML. In AML patients, circulating $\gamma \delta$ T-cells $[6,7]$ which may be expanded ex vivo using the synthetic PAg, Phosphostim. Since Phosphostim is no longer undergoing clinical development, we evaluated an alternative system comprising zoledronic acid, IL-2 and IL-15 [8]. We have previously shown that this cocktail can expand $\gamma \delta$ T-cells from patients with ovarian cancer [8] and hypothesized that a similar approach could be applied in AML patients.

\section{Methods}

\section{Processing of peripheral blood samples}

Seventeen blood samples (30 mL each) derived from 10 AML patients (clinical data - Table 1) were provided by the King's College London Haemato-Oncology Tissue Bank (Human Tissue Authority license 12223; National Research Ethics reference 08/H0906/94). All patient-derived peripheral blood samples were independently analyzed for the presence of circulating AML blasts by morphology alone, or accompa- nied by flow cytometry at the time of banking. Blood samples were also obtained from healthy donors under approval of the South East London Research Ethics Committee 1 (09/ $\mathrm{H} 0804 / 92)$.

\section{Culture of primary human $\gamma \delta$ T-cells}

Ficoll-separated PBMC were cultured at $3 \times 10^{6} \mathrm{cells} / \mathrm{ml}$ in RPMI 1640 (Lonza, Basel, Switzerland), 10\% human AB serum (Sigma, Poole, UK), GlutaMax (Life Technologies, Paisley, UK) and antibiotic-antimycotic solution (Life Technologies). On day 1 , zoledronic acid ( $1 \mu \mathrm{g} / \mathrm{ml}$; Zometa, Novartis, Frimley/ Camberley, UK), IL-2 (100 U/ml; Proleukin, Novartis) and IL15 (10 ng/ml; Gentaur, Kampenhout, Belgium) were added. Additional medium and cytokines were added every 2-3 day for 15 days. Numbers of $\gamma \delta$ T-cells were determined manually as total cell number multiplied by percentage $\gamma \delta$ T-cells in the PBMC gate/100.

\section{Flow cytometry}

Flow cytometric analysis was undertaken on freshly isolated PBMC and ex vivo expanded $\gamma \delta$ T-cell cultures as described [8], using antibodies listed in Supplementary Table 1. Analysis was performed using a FACScalibur cytometer with Cellquest Pro software. Representative forward scatter versus side 
Citation: Parente-Pereira AC, Krishnamurthy P, Whilding LM, et al. (2020) Efficient Ex Vivo Expansion of $ү \delta$ T-Cells from AML Patients Requires Elimination of Circulating Leukemic Blasts. Advances Leuk Res Treat 2(1):28-33

scatter gating strategies on freshly isolated PBMC are shown (Supplementary Figure 1).

\section{Cytotoxicity assays}

PKH-labeled KG-1 leukemic cells were incubated \pm zoledronic acid $(5 \mu \mathrm{g} / \mathrm{ml})$ or alendronic acid $(20 \mu \mathrm{g} / \mathrm{ml})$ overnight before co-culture with $\gamma \delta$ T-cells at the indicated effector:target ratio for 4 hours. Apoptotic cells were detected after Annexin $\mathrm{V}$ staining by flow cytometry (Supplementary Figure 2) [8].

\section{Enzyme-linked immunosorbent assay}

Supernatants were analyzed using a human IFN- $\gamma$ enzyme-linked immunosorbent assay (ELISA; Ready-set-go kit
(eBiosciences, Hatfield, UK)) as described by the manufacturers.

\section{Statistical analysis}

Statistical analysis was performed using One-way ANOVA for analysis of groups of 3 or more datasets. Alternatively, an unpaired two-tailed Student's $t$ test or Mann-Whitney test was used to compare two groups, where data passed or failed normality testing respectively. All statistical analyses were performed using GraphPad Prism version 5.0, 6.0 or 7.0 (GraphPad software, San Diego, CA, USA).

\section{Results and Discussion}

\section{Expansion of $\gamma \delta$ T-cells from $A M L$ patients}

B

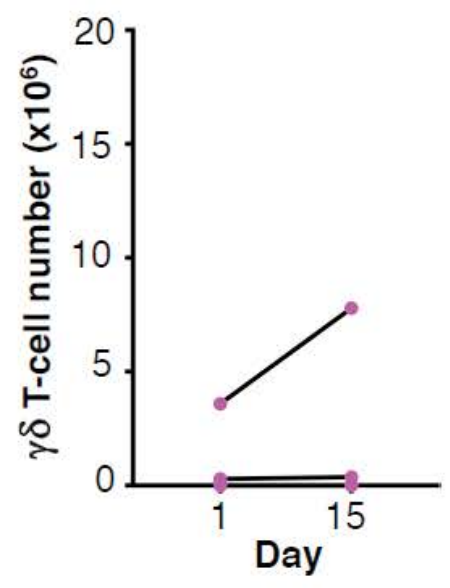

C

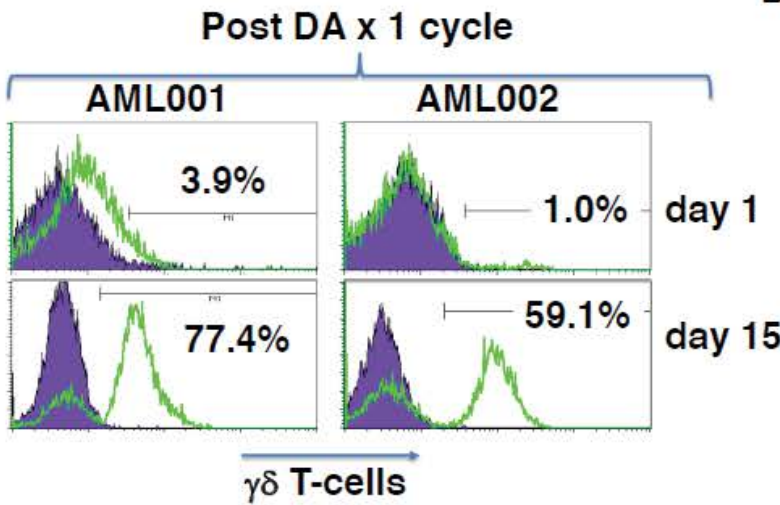

Figure 1: Ex vivo expansion of $\gamma \delta$ T-cells from AML patients with circulating blast cells is inefficient. (A) PBMC were separated from two patients with newly diagnosed AML (AML001 - M1 subtype without FLT3 internal tandem duplication (ITD); AML002 - M1 with FLT3 ITD and NPM1 mutation) in whom circulating blast cells were present. Samples were stained with a pan- $\gamma \delta$ TCR antibody (open green histogram) and analyzed by flow cytometry, making comparison with an isotype control antibody (filled purple histogram) (day 1). Cultures were then stimulated with ZA followed by IL-2/IL-15 for 15 days prior to re-analysis using similar methodology; (B) Pooled data from 5 AML patients in whom circulating leukemic blasts were present at the time of PBMC collection. In each case, $\gamma \delta$ T-cell number was determined manually before (day $1-30 \mathrm{~mL}$ blood sample) or after culture of derived PBMC for 15 days in zoledronic acid + IL-2/IL-15; (C) Following one cycle of daunorubicin plus cytarabine (DA) induction chemotherapy, PBMC from patients AML001 and AML002 were re-analyzed for $\gamma \delta$ T-cells by flow cytometry, both before and after culture in zoledronic acid + IL-2/IL-15; (D) Following 1 cycle of fludarabine plus cytarabine (FLA) chemotherapy, PBMC were separated from AML003 (AML derived from JAK2 mutant myeloproliferative disorder) and were analyzed by flow cytometry before and after culture in zoledronic acid + IL-2/IL-15, as described above. Twenty nine days later, relapse occurred accompanied by circulating blasts and a second sample was analyzed similarly. 
A

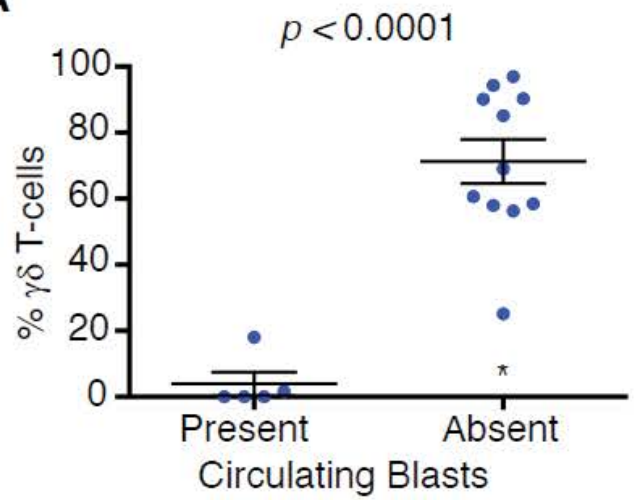

B

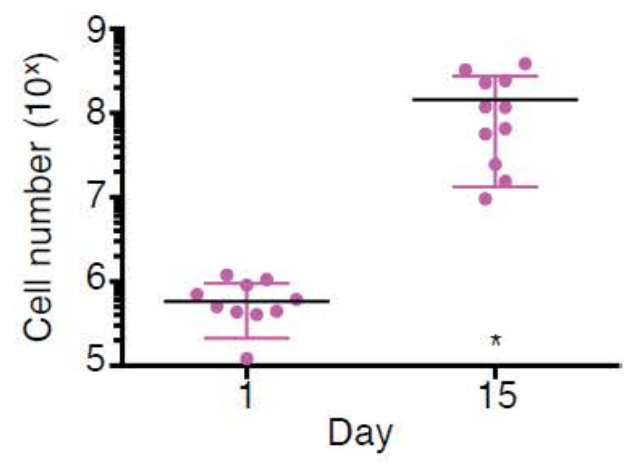

D
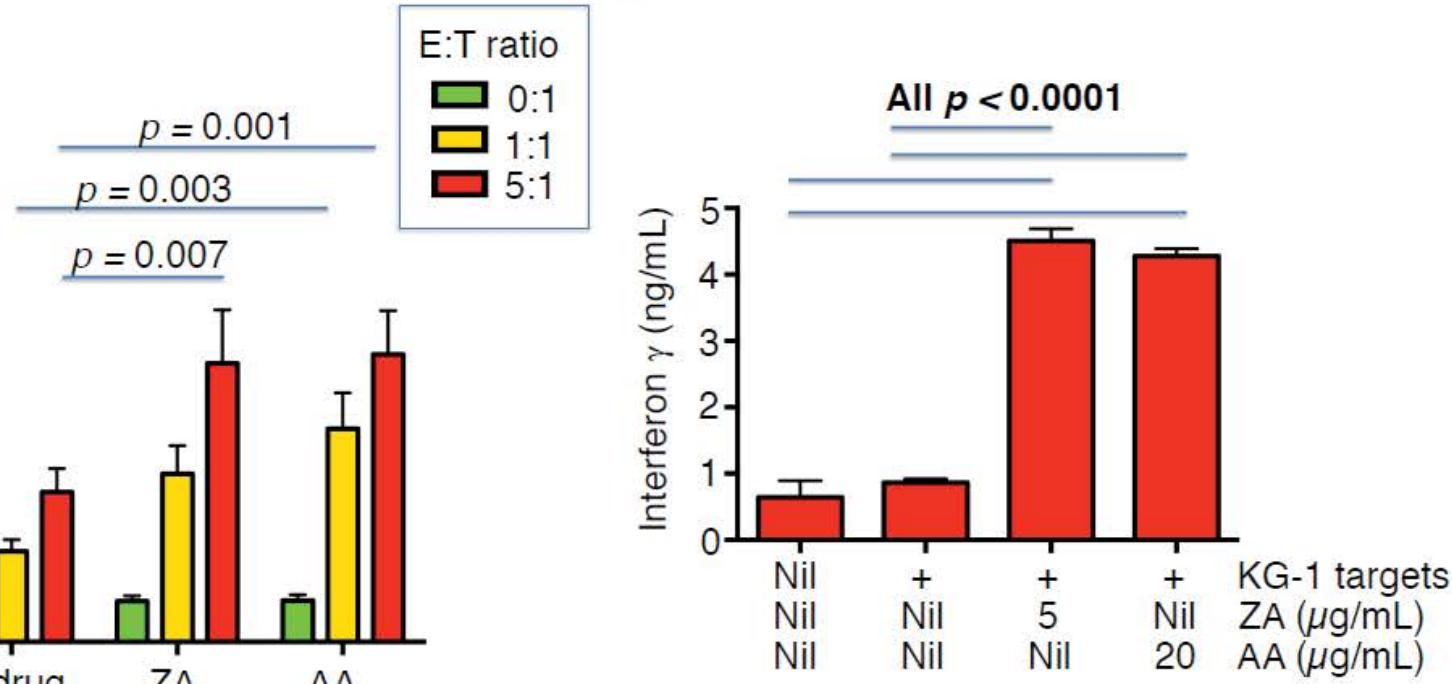

Figure 2: Characterization of $\gamma \delta$ T-cells expanded from patients with newly diagnosed AML following chemotherapy-mediated clearance of circulating blast cells. (A) Seventeen peripheral blood samples were obtained from 10 AML patients with $(n=5)$ or without $(n=12)$ circulating blasts were cultured in zoledronic acid (ZA) + IL-2/ IL-15. Percentage $\gamma \delta$ T-cells in the culture was analyzed after 15 days; (B) Total number of $\gamma \delta$ T-cells present in the 11 samples that were free of circulating AML blasts and contained detectable CD3 ${ }^{+} \mathrm{T}$-cells is shown before and after culture in ZA + IL-2/IL-15 for 15 days. ${ }^{*}$ One sample contained an absolute lymphocyte count of $0.2 \times 10^{\circ} / \mathrm{L}$ within which no $\mathrm{CD}^{+}$cells were detectable and has been omitted; (C) PKH red fluorescent labeled KG-1 leukemic cells were incubated \pm ZA or alendronic acid (AA) overnight before co-culture with $\gamma \delta$ T-cells at the indicated effector:target ratio for 4 hours. Apoptotic cells were detected after Annexin V-FITC staining by flow cytometry (mean \pm SEM; $\gamma \delta$ T-cells expanded from 8-10 independent donors); (D) Ex vivo expanded $\gamma \delta$ T-cells (sample 2 from patient 007) were co-cultivated for 24 hours at 1:1 effector:target ratio with KG-1 leukemic cells. Where indicated, KG-1 target cultures had been pre-incubated overnight with zoledronic or alendronic acid prior to addition of $\gamma \delta$ T-cells. Supernatants were harvested and analyzed for IFN- $\gamma$ (mean \pm SEM, triplicate cultures). Statistical significance was analyzed using One-way ANOVA.

We explored the feasibility of zoledronic acid-mediated $\delta$ T-cell expansion from patients with AML. However, only a relatively small percentage of $\gamma \delta$ T-cells could be identified in ficoll-separated PBMC derived from newly diagnosed $A M L$ patients (representative examples - Figure 1A). Furthermore, zoledronic acid-mediated expansion of these cells was either inefficient or unsuccessful in each of five consecutive attempts (Figure 1B).

Patients with AML who achieve minimal residual disease status following chemotherapy are reported to have increased circulating $\gamma \delta$ T-cell numbers. By contrast, these cells are substantially reduced in patients with high disease burden [6]. Consequently, we hypothesized that disease status might be responsible for poor zoledronic acid-mediated $\gamma \delta$ T-cell expansion. To test this, we obtained further blood samples from two of the previously described AML patients (AML001 and AML002), following induction chemotherapy with a single cycle of daunorubicin and cytarabine (DA) chemotherapy. In both cases, a discrete population of $\gamma \delta$ T-cells was now evident in isolated PBMC (Figure $1 C$ ). Moreover, $\gamma \delta$ T-cells expanded efficiently following zoledronic acid-mediated activation (Figure $1 \mathrm{C}$ ), contrasting with the failure of expansion observed prior to chemotherapy. Conversely, sequential samples were also obtained from a third patient (AML003), immediately following FLA chemotherapy (fludarabine/cytarabine), when circulating blasts were undetectable, or following subsequent disease relapse. In this case, a clearly distinct $\gamma \delta$ T-cell population was only detected in the leukemia-free sam- 
Citation: Parente-Pereira AC, Krishnamurthy P, Whilding LM, et al. (2020) Efficient Ex Vivo Expansion of $\gamma \delta$ T-Cells from AML Patients Requires Elimination of Circulating Leukemic Blasts. Advances Leuk Res Treat 2(1):28-33

ple and these cells alone were amenable to efficient expansion by ZA (Figure 1D).

\section{Characterization of circulating and ex vivo ex- panded $\gamma \delta$ T-cells from AML patients}

These preliminary findings suggested that it would be more profitable to expand circulating $\gamma \delta$ T-cells from peripheral blood in which circulating leukemic blasts were absent following chemotherapy. In such AML patients, $\gamma \delta$ T-cells accounted for $1.55 \pm 3.13 \%$ of PBMC $(19,388 \pm 12,302 \gamma \delta$ cells/ $\mathrm{ml}$ blood; mean $\pm S D, n=11$ ), which was not significantly different to healthy donors $(19,916 \pm 29,887$, mean $\pm S D, n=21)$ [8]. In all cases but one, these $\gamma \delta$ T-cells were amenable to zoledronic acid-mediated enrichment, unlike those from patients with circulating leukemic cells (Figure 2A). In the single case that expansion failed, there were no $\mathrm{CD}^{+} \mathrm{T}$-cells detectable (Table 1).

Next, we analyzed the expansion and immunophenotype of zoledronic acid-expanded $\gamma \delta$ T-cells following chemotherapy-mediated clearance of circulating blasts. We observed that these cells expanded by an average of 229-fold (Figure 2B), which is similar to healthy donors [8]. Expanded cells exhibited high levels of CD27, CD62L and CCR7 and lower expression of CD70 (Supplementary Figure 3 and Supplementary Figure 4), consistent with the presence of central memory cells $[9,10]$.

Expression of NKG2D also plays an important role in the ability of $\gamma \delta$ T-cells to recognize stress ligands on AML blasts [11], although recent data has raised doubts about the expression of such ligands on the stem cell compartment [12]. Cells expanded from AML patients post-chemotherapy expressed comparable high levels of NKG2D to cells derived from healthy donors (Supplementary Figures 3 and Supplementary Figure 4). Furthermore, these cells consistently exhibited anti-leukemic cytotoxic activity in a manner that was potentiated by bisphosphonate sensitization (Figure $2 \mathrm{C}$ and Supplementary Figure 1) and accompanied by IFN- $\gamma$ release (Figure 2D).

\section{Conclusions}

The major finding of this short communication is that functional $\gamma \delta$ T-cells can only be expanded efficiently from AML patients after chemotherapy-mediated clearance of circulating leukemic cells. Mechanistically, this may reflect the uncoupling of the multiple immunosuppressive mechanisms that are found in newly diagnosed or relapsed AML. Leukemic blasts have been compared to myeloid-derived suppressor cells. In active disease, there is also an increase in regulatory T-cells, immunosuppressive exosomes and inhibitory molecules such as CD200, PD-L1 and indoleamine dioxygenase [1320]. Future studies should undertaking mixing/depletion experiments with AML cells, analysing effects on $\gamma \delta$ T-cell numbers, phenotype and exhaustion markers. Furthermore, in planning for immunotherapy of AML with autologous ex vivo expanded $\gamma \delta \mathrm{T}$-cells, our data suggest that harvest of starting material should be scheduled when disease is in remission. This is logical since disease burden and circulating blasts are minimal at this time while $\gamma \delta$ T-cells are most amenable to expansion, while retaining anti-leukemic activity.

\section{Funding}

This work was supported by Bloodwise [programme 13007]; the Experimental Cancer Medicine Centre at King's College London and the National Institute for Health Research (NIHR) Biomedical Research Centre based at Guy's and St Thomas' National Health Service (NHS) Foundation Trust and King's College London. The views expressed are those of the authors and not necessarily those of the NHS, the NIHR or the Department of Health.

\section{Declaration of Competing Interests}

J.M. is a founding scientist of Leucid Bio. Other authors declare no competing financial interests.

\section{Acknowledgements}

We thank members of the King's College London Haemato-Oncology Tissue Bank for provision of blood samples.

\section{References}

1. HJ Gober, M Kistowska, L Angman, et al. (2003) Human T cell receptor gammadelta cells recognize endogenous mevalonate metabolites in tumor cells. J Exp Med 197: 163-168.

2. A Pandyra, PJ Mullen, M Kalkat, et al. (2014) Immediate utility of two approved agents to target both the metabolic mevalonate pathway and its restorative feedback loop. Cancer Res 74: 47724782.

3. HY Li, FR Appelbaum, CL Willman, et al. (2003) Cholesterol-modulating agents kill acute myeloid leukemia cells and sensitize them to therapeutics by blocking adaptive cholesterol responses. Blood 101: 3628-3634.

4. M Fiegl, M Juergens, W Hiddemann, et al. (2007) Cytotoxic activity of the third-generation bisphosphonate zoledronic acid in acute myeloid leukemia. Leuk Res 31: 531-539.

5. L Halim, AC Parente-Pereira, J Maher (2017) Prospects for immunotherapy of acute myeloid leukemia using gammadelta T cells. Immunotherapy 9: 111-114.

6. JM Aswald, XH Wang, S Aswald, et al. (2006) Flow cytometric assessment of autologous gammadelta $T$ cells in patients with acute myeloid leukemia: potential effector cells for immunotherapy?. Cytometry B Clin Cytom 70: 379-390.

7. J Gertner-Dardenne, R Castellano, E Mamessier, et al. (2012) Human Vgamma9Vdelta2 $T$ cells specifically recognize and kill acute myeloid leukemic blasts. J Immunol 188: 4701-4708.

8. AC Parente-Pereira, H Shmeeda, LM Whilding, et al. (2014) Adoptive immunotherapy of epithelial ovarian cancer with Vgamma9Vdelta2 $\mathrm{T}$ cells, potentiated by liposomal alendronic acid. J Immunol 193: 5557-5566.

9. M Eberl, R Engel, E Beck, et al. (2002) Differentiation of human gamma-delta $T$ cells towards distinct memory phenotypes. Cell Immunol 218: 1-6.

10. F Dieli, F Poccia, M Lipp, et al. (2003) Differentiation of effector/ memory Vdelta2 $\mathrm{T}$ cells and migratory routes in lymph nodes or inflammatory sites. J Exp Med 198: 391-397.

11. S Gundermann, E Klinker, B Kimmel, et al. (2014) A comprehensive analysis of primary acute myeloid leukemia identifies bio- 
markers predicting susceptibility to human allogeneic Vgamma9Vdelta2 T cells. J Immunother 37: 321-330.

12. AM Paczulla, K Rothfelder, S Raffel, et al. (2019) Absence of NKG2D ligands defines leukaemia stem cells and mediates their immune evasion. Nature 572: 254-259.

13. Q Zhou, ME Munger, SL Highfill, et al. (2010) Program death-1 signaling and regulatory $T$ cells collaborate to resist the function of adoptively transferred cytotoxic $T$ lymphocytes in advanced acute myeloid leukemia. Blood 116: 2484-2493.

14. T Tian, S Yu, L Liu, et al. (2015) The Profile of T Helper Subsets in Bone Marrow Microenvironment Is Distinct for Different Stages of Acute Myeloid Leukemia Patients and Chemotherapy Partly Ameliorates These Variations. PLoS One 10: e0131761.

15. SJ Coles, MN Gilmour, R Reid, et al. (2015) The immunosuppressive ligands PD-L1 and CD200 are linked in AML T-cell immunosuppression: identification of a new immunotherapeutic synapse. Leukemia 29: 1952-1954.
16. MJ Szczepanski, M Szajnik, M Czystowska, et al. (2009) Increased frequency and suppression by regulatory $T$ cells in patients with acute myelogenous leukemia. Clin Cancer Res 15: 3325-3332.

17. A Curti, S Trabanelli, C Onofri, et al. (2010) Indoleamine 2,3-dioxygenase-expressing leukemic dendritic cells impair a leukemia-specific immune response by inducing potent $\mathrm{T}$ regulatory cells. Haematologica 95: 2022-2030.

18. T Hoeres, E Holzmann, M Smetak, et al. (2019) PD-1 signaling modulates interferon-gamma production by Gamma Delta (gammadelta) T-Cells in response to leukemia. Oncoimmunology 8: 1550618.

19. CS Hong, P Sharma, SS Yerneni, et al. (2017) Circulating exosomes carrying an immunosuppressive cargo interfere with cellular immunotherapy in acute myeloid leukemia. Sci Rep 7: 14684.

20. AJ Barrett (2020) Acute myeloid leukaemia and the immune system: implications for immunotherapy. Br J Haematol 188: 147158. 